\title{
A New Watermarking Algorithm for Point Model Using Angle Quantization Index Modulation
}

\author{
Feng Xiaoqing \\ Institute of Computer Application Techniques, Zhejiang University of Finance \& Economics, China \\ e-mail: zllinda13@sohu.com
}

Keywords: point cloud; Angle Quantization Index Modulation; PCA

\begin{abstract}
Point cloud models are widely used in industry. It is also faced with increasingly serious copyright protection issues. A new watermarking algorithm of 3D point cloud model using angle quantization index modulation is proposed. At first, a point cloud model is segmented into many patches according to the length of watermark information. Then, for each patch, 1 bit watermark is embedded into by modulating the angle of feature signal. Experiments show that the algorithm is certain robustness against affine transformation, reorder and low intensity of noise.
\end{abstract}

\section{Introduction}

Digital watermarking by inserting some invisible information is an effective method to protect copyright of digital media. People mainly focused on images [1] and videos [2]. And few works have been done on point model. However, point cloud model can express a complicated object only by point information. This character makes point cloud used widely in fields of computer graphic. It is becoming urgent to research a practical watermark algorithm for point clouds.

So far, several point cloud watermark schemes have been developed. A fast hierarchical clustering algorithm is applied to partition patches by Cott [3]. Then, Laplacian eigen-functions of each patch are achieved to insert watermark. It is robust against attacks, including affine transformations, cropping, noising and resampling. Ohbuchi et al. extend a mesh watermarking algorithm to point models [4]. So, it need generates a non-manifold mesh for each cluster of the points. Then, wavelet-based spectral analysis technique proposed by Zachi Karni[5] is applied to insert into watermark. Two watermark algorithms in above are non-blind watermark because original point model is need provided during extraction. Zhu proposed an improved AQIM algorithm [6], and prove the effectiveness of the scheme on images. In this paper, we extend this image AQIM algorithm to 3D point cloud models. Because there is not inherent order to show point cloud like image from left to right, a point cloud model should be sorted in a certain order. Furthermore, for decreasing the distortion causing by embedding watermark into model, a point model need to divided into many groups according to the numbers of watermark bit. For each group, 1 bit watermark are embedded into by an angle quantization index modulation scheme.

The remainder of this paper is organized as follows. In Section 2, related work is described. An improved AQIM watermarking is described in detail in Section 3. The performance of algorithm is shown in section 4. Finally the future work is looked forward in Section 5.

\section{Related works}

\section{Watermarking algorithms based on QIM}

The QIM scheme proposed by Chen et al. [7] modulate the host signal to its closest code words according to the watermark bit, as shown in figure 1. Dither modulation (DM) is a kind of basic implementation of QIM. This algorithm can keep a better balance between imperceptible, watermark capacity and robustness. So, QIM algorithm is widely used in image watermarking scheme. 


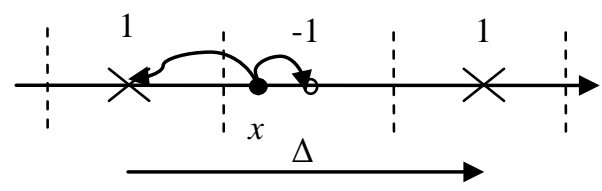

Figure 1 Principle of QIM

However, the QIM watermarking method does not resist against some attacks, for example, compensation and scaling. For improving the performance of watermarking, a lot of efforts have been done in terms of choosing a suitable quantization feature. Thus, many improved methods based on QIM have been proposed, such as the spread-transform dither modulation (STDM) [8], the logarithmic QIM (LQIM) [9], and the angle QIM (AQIM) method [10]. STDM is as an extension of $\mathrm{DM}$ in [7], projection feature is quantized along a random direction, which makes STDM has significant advantages. Logarithmic transform and non-uniform quantization step are used in LQIM, which makes it can resist against scaling attack. AQIM first introduced in [10] is an extension of the QIM to angle. The different with traditional QIM method, the angle is quantized instead of quantizing the amplitude values. The angle of vector is changed to the most closed codeline according to embedded watermark bit as shown in Figure. 2.

AQIM algorithm can be simplified described as following:

1) The angle of the host vector is quantized according to quantizers $Q(\cdot)$.

$$
Q(\theta)=\Delta \cdot\left\lfloor\frac{\theta+m \frac{\Delta}{2}}{\Delta}\right\rfloor+m \cdot \frac{\Delta}{2}
$$

2) Change the angle of the vector to closest code words to obtain the watermarked vector.

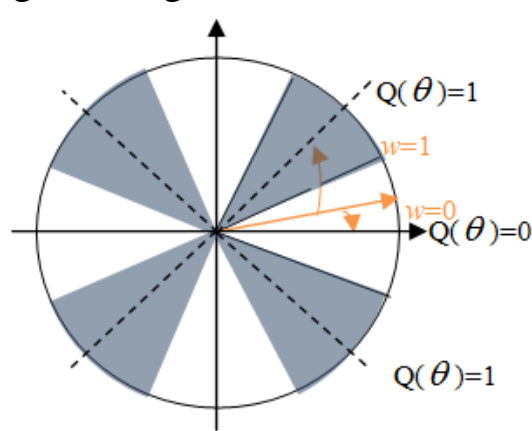

Figure 2 AQIM algorithm

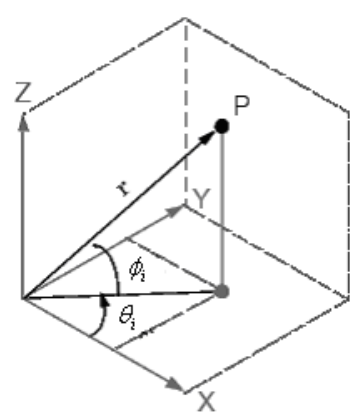

Figure. 3 The relationship between $\mathrm{x}, \mathrm{y}, \mathrm{z}$ and $\mathrm{r}, \theta, \varphi$

Although the types of a carrier signal are not any restricted in QIM, they are not directly applied to 3D model. Darazi [11] developed a sparse QIM for 3D mesh models. The scheme of quantization the distance of vertex in the spherical coordinate system makes the algorithm robust to affine transformation. In our QIM of 3D point model, we adopt Darazi's scheme to against affine transformation.

\section{The watermarking algorithm}

We are inspired by papers [6,10]. And we try to apply this algorithm [6] to 3D point cloud model. In this section, a novel watermarking algorithm for point cloud model based on AQIM will be descried in detail.

The block diagram of this method is shown in Figure 4. 


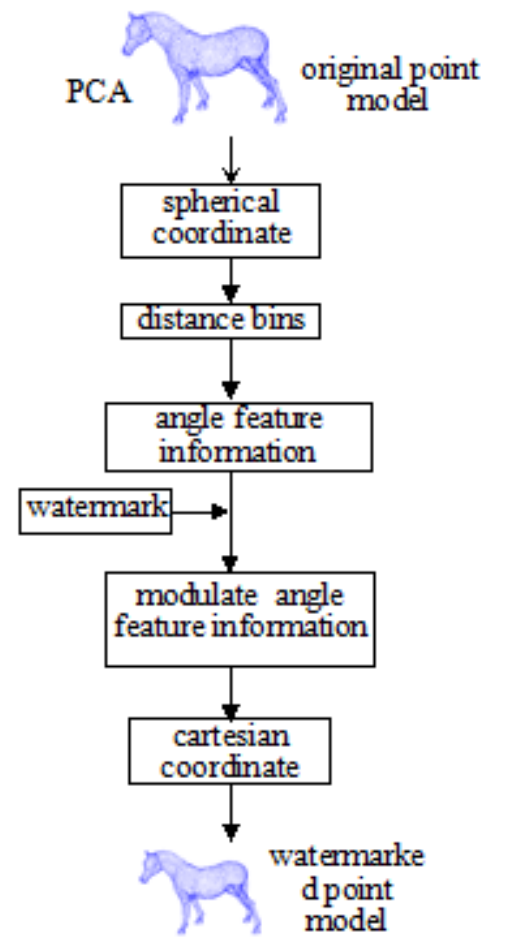

(a) Watermark embedding

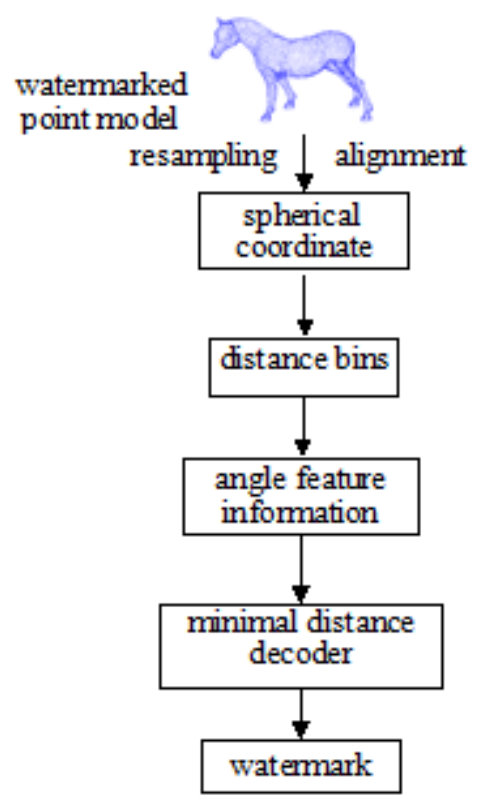

(b) Watermark extraction

Figure 4 The block diagram of the watermark algorithm

A new coordinate system of a point cloud model is constructed by PCA, and then, the Cartesian coordinates $v_{i}=\left(x_{i}, y_{i}, z_{i}\right)$ is converted into the spherical coordinates $q_{i}=\left(r_{i}, \theta_{i}, \varphi_{i}\right)$, as shown in figure 3.

A distance set $D=\left\{r_{i} \mid r_{1}, r_{2}, \ldots . r_{n}, r_{i}<r_{i+1}\right\}$ is divided into some bins according to the length of watermark. For each group, we calculate the angle $\theta_{m}=\arccos \frac{\mathbf{R}_{m} \mathbf{u}}{\left\|\mathbf{R}_{m}\right\|\|\mathbf{u}\|}$. Here, $\mathbf{R}=\left[\begin{array}{c}R_{1} \\ \ldots \\ R_{m}\end{array}\right]=\left[\begin{array}{cccc}r_{1} & r_{2} & \ldots & r_{j} \\ & \ldots & \\ r_{p+1} & r_{2} & \ldots & r_{q}\end{array}\right], j=\left\lfloor\frac{n}{n_{w}}\right\rfloor, q=\left\lfloor\frac{n}{n_{w}}\right\rfloor \cdot n_{w}$.

In our algorithm, watermarking is embedded by modulating the angle of $\theta_{m}$. Two quantizers $Q_{0}(\cdot)$ and $Q_{1}(\cdot)$ are constructed, $Q_{0}(\mathrm{Z}, \Delta)=\left[\frac{\mathrm{Z}}{\Delta}-\frac{d}{\Delta}\right] \Delta+d$ and $Q_{1}(\mathrm{Z}, \Delta)=\left[\frac{\mathrm{Z}}{\Delta}-\frac{1}{2}-\frac{d}{\Delta}\right] \Delta+\frac{1}{2} \Delta+d$.

The signal of $\theta_{m}$ is quantized by choosing one of quantizers, $\theta_{w}=Q_{w}\left(\theta_{m}, \Delta\right), w \in\{-1,1\}$. If $\theta_{w} \notin[-1,1], \theta_{w}=\theta_{w} \pm \Delta$

In our embedding, the step size $\Delta=0.002$, which is an empirical value. Futhermore, watermarked angle signal $A$ is required to satisfy a condition of $\theta_{A}=\theta_{w}$, where $\theta_{A}$ has the same definition as $\theta_{R}$. The embedding region is the surface of a circular cone with the axis $u$ and the angle $2 \theta_{A}$. For getting the minimize distortion causing by embedding, the embedding function is considered as $\theta_{R}$ project on the cone.

Paper [6] descirbe the geometric interpretation of watermark embedding function. Aaccording to [6], $R_{w}=R^{T} R_{w d} R_{w d}$ and $R_{w d}=\lambda R_{d}+\mu X_{d}$ can be achieved. Moreover, the value of $R_{w d}$ satisfies the conditions $R_{w d}^{T} R_{w d}=1$ and $R_{w d}^{T} X_{d}=\cos \left(\theta_{w}\right)$. According to these analysis in above, 
$\lambda=\sqrt{\frac{1-\cos ^{2}\left(\theta_{w}\right)}{1-\cos ^{2}\left(\theta_{R}\right)}}$ and $\mu=\cos \left(\theta_{w}\right)-\lambda \cos \left(\theta_{R}\right)$ can be calculated. At last, we will get the watermarked signal $\mathbf{R}_{w}, \mathbf{R}_{w}=\left[\begin{array}{llll}r_{w 1} & r_{w 2} & \ldots & r_{w n_{b i t}}\end{array}\right]^{T}$.

\section{Watermark extracted}

The process of extraction algorithm is alike to embed process. For achieving the sort order of the original model, resampling operation must be done. The angle of attacked signal $f_{A}$ is calculated. At last, a watermark $\widehat{w}$ is extracted by the minimal distance decoder, $\widehat{w}=\arg \min _{m \in\{-1,1\}}\left|\theta_{A}-Q_{m}\left(\theta_{A}\right)\right|$. The bit error rate (BER) is used for judging whether there are watermark embedded in a point model .

\section{Simulation results}

Embedding watermark will cause geometrical distortion of a point model. HausdorffDist is a common evaluating indicator. So, we use HausdorffDist [12] of $r_{i}$ between original and watermarked point model.

Table 1 provides information of tested point cloud models. In the simulations, a 64 bits of watermark is embedded into a point model.

Table 1 Point cloud models

\begin{tabular}{|c|c|c|}
\hline $\begin{array}{c}\text { Original } \\
\text { Model }\end{array}$ & (23984 points $)$ \\
\hline $\begin{array}{c}\text { WewVenus } \\
\text { Watermarked } \\
\text { Model }\end{array}$ & & \\
\hline HausdorffDist & 0.1240 & 0.0173 \\
\hline
\end{tabular}

1) Affine transformation

Affine transformation includes translation, scaling and rotation, which are a common operation rather than any of attacks. Thus, robustness against these operations is very important for a reversible watermark scheme. The simulation experiments of similarity transformation are shown in Table 2.

2) Reordering

Distances between points and the center of model is sorted in ascending order, watermark can be integrality extracted after model is attacked by reordering.

3) Noising

Binary random noise is added to each vertex of line entities with different error rates on the model. The experiment results of noising attacks are shown in Table 3 and figure 5. 


\section{Conclusion}

In this new watermarking scheme based on AQIM, a point model is divided into many patches according to the numbers of watermarking bit. Then, 1 bit watermark information is embedded into a patch. The experimental results show that the introduced algorithm is certain robust against some attacks, such as affine transformation, reorder and low intensity of noise. However, we find that BERs will become greater with the increasing of noise intensity.

Table 2 Experiment results

\begin{tabular}{|c|c|c|c|c|}
\hline \multicolumn{2}{|c|}{$\begin{array}{l}\text { Model } \\
\text { Type attacks }\end{array}$} & NewVenus & fo1 & miscDINO \\
\hline \multicolumn{2}{|c|}{$\begin{array}{c}\text { similarity } \\
\text { transformation }\end{array}$} & 0 & 0 & 0 \\
\hline \multicolumn{2}{|c|}{ re-order } & 0 & 0 & 0 \\
\hline \multirow{4}{*}{$\begin{array}{l}\text { strength of } \\
\text { noising }\end{array}$} & $0.25 \%$ & $1 / 64$ & 0 & 0 \\
\hline & $0.5 \%$ & $13 / 64$ & $9 / 64$ & $5 / 64$ \\
\hline & $2 \%$ & $26 / 64$ & $31 / 64$ & $34 / 64$ \\
\hline & $5 \%$ & $28 / 64$ & $28 / 64$ & $39 / 64$ \\
\hline
\end{tabular}

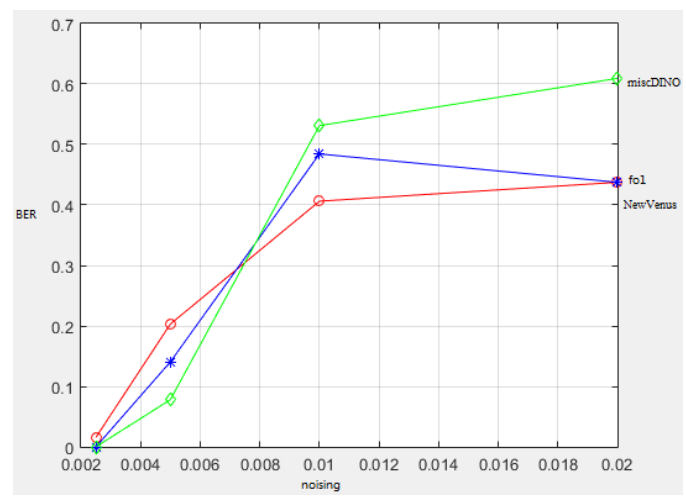

Figure 5 Noising attack

\section{Acknowledgment}

This work is supported by the National Natural Science Foundation of China (No.61202197).

\section{References}

[1] E. Nezhadarya, Z. J. Wang, R. K. Ward, "Robust image watermarking based on multiscale gradient direction quantization". IEEE Trans. Inf. Forensics Security, 6( 4), (2011), 1200-1213.

[2] H. Y. Chen, Y. S. Zhu,"A robust video watermarking algorithm based on singular value decomposition and slope-based embedding technique". Multimed Tools App. DOI 10.1007/s11042-012-1238-2, 2012

[3] D. Cotting, T. Weyrich , M. Pauly, M. Gross, "Robust Watermarking of Point Sampled Geometry". In: the Proceedings of the international Conference on Shape Modeling and Application, Italy, 2004: 233-242

[4] Ohbuchi R., Mukaiyama A., Takahashi S. "Watermarking a 3D shape model defined as a point set". In: Proceedings of the International Conference on Cyber worlds (CW04), Tokyo, 2004, 392-399

[5] S. Kanai, H. Date, T. Kishinami, "Digital watermarking for 3D polygons using multiresolution wavelet decomposition". In: Proceedings of the 6th IFIP WG 5.2 International Workshop on Geometric Modeling, Tokyo, 1998, 296-307 B. Chen and G. W. Wornell, "Quantization index 
modulation: a class of provably good methods for digital watermarking and information embedding". IEEE Trans. Inf. Theory, 47(4), (2001), 1423-1443.

[6] X. S. Zhu, S. L. Pen, "Novel Quantization Watermarking Based on Random Angle Modulation". In: 2011 Seventh International Conference on Computational Intelligence and Security. 16(7), (2011), 959-963,

[7] B. Chen, and G. W. Wornell, "Preprocessed and post processed quantization index modulation methods for migital watermarking", Proc. of the SPIE International Conference on Security and Watermarking of Multimedia, (2000), 48-59.

[8] B. Chen, and G. W. Wornell, "Quantization index modulation: a class of provably good methods for digital watermarking and information embedding", Information Theory IEEE Transactions on, 47(4), (2001), 1423-1443.

[9] N. K. Kalantari, and S. M. Ahadi, "A logarithmic quantization index modulation for perceptually better data hiding", IEEE transactions on image processing : a publication of the IEEE Signal Processing Society, 19(6), (2010), 1504-1517

[10]F. Our'1que, V. Licks, R. Jordan, and F. P' erez-Gonz` alez, "Angle qim :a novel watermark embedding scheme robust against amplitude scaling distortions". In Proc. Int. Conf. Acoustics, Speech and Signal Processing, (2005), 797-800.

[11]R. Darazi, R. Hu, and B. Macq, "Applying spread transform dither modulation for 3D-mesh watermarking by using perceptual models", In Proc. IEEE Int. Conf. Acoust., Speech, and Signal Processing (ICASSP'10), Mar. 19, (2010),1742-1745

[12]http://www.mathworks.com/matlabcentral/fileexchange/26738-hausdorff-distance 